Annals of Pure and Applied Mathematics

Vol. 18, No. 2, 2018, 213-217

ISSN: 2279-087X (P), 2279-0888(online)

Published on 31 December 2018

www.researchmathsci.org

DOI: http://dx.doi.org/10.22457/apam.v18n2a12

Annals of

Pure and Applied

Mathematics

\title{
Solution of the Erdös-Moser Equation
}

$$
1+2^{p}+3^{p}+\cdots+k^{p}=(k+1)^{p}
$$

David Stacha

Obchodna 32, 90638 Rohoznik,Slovak Republik

E-mail: safiro@centrum.sk

Received 30 November 2018; accepted 28 December 2018

"To solve a mathematical problem does not mean to discover something new, it means to understand the connections as old as the universe itself."

David Stacha

Abstract. The principal aim of this paper is to provide a solution of the ErdösMoser equation, based on the properties of Bernoulli polynomials, and prove that there is only one solution satisfying the above-mentioned equation.

Keywords: Bernoulli polynomials, Summation, Diophantine equation

AMS Mathematics Subject Classification (2010): 11D41, 11D72, 11B68

\section{Notation}

$1+2^{p}+3^{p}+\cdots+k^{p}=(k+1)^{p}$ represents the Erdös-Moser equation, where

$k, p \in \mathbb{N}^{*}$. Let $b_{n}$ denote Bernoulli numbers and $B_{n}(x)=\sum_{k=0}^{n}\left(\begin{array}{l}n \\ k\end{array}\right) b_{n-k} x^{k}$ denote Bernoulli polynomials for $n \geq 0$.

\section{Introduction}

The Erdös-Moser equation (EM equation), named after famous mathematicians Paul Erdös and Leo Moser, represents an exponential Diophantine equation. Moreover, this equation differs from any other Diophantine equation since combines addition, powers and summation together. These properties make the equation even more interesting, and therefore it has been studied by many number theorist throughout history. The open and very fascinating conjecture of ErdösMoser states that there is no other solution of the EM equation than trivial $1+2=3$. So far, there have been only partial results defining the lower bound of $k$ for which the EM equation could have another solution, but a complete solution had been missing. In general, Diophantine equations are widely studied object in mathematics, mainly in number theory. The unknowns of these equations take only integer values, and exactly this restriction makes them interesting on the one hand, and on the other hand much more difficult. This is also the case of the EM 


\section{David Stacha}

equation because if we would solve this equation, where $p \in \mathbb{R}$, there would be infinitely many solutions, but only one restriction, namely an adjective Diophantine, has a big impact on the number of solutions and causes that infinitely many solutions of the EM equation will be reduced to only one. Moreover, if any Diophantine equation is solved in $\mathbb{N}^{*}$, as in our case, the equation can be related to some problem including counting and ordering. Aiming for a proof, an investigation of the properties and identities of the EM equation will be discussed in the following sections.

\section{Solution}

Lemma 3.1. The EM equation is equivalent to

$$
\sum_{k=0}^{x} k^{p} \equiv \frac{B_{p+1}(x+1)}{p+1}=(x+1)^{p}
$$

$x, p \in \mathbb{N} \wedge x>2 \wedge p>1$ since we are seeking other solution than trivial.

Proof: Sum of pth powers is defined as

$$
\sum_{k=0}^{x} k^{p}=\frac{B_{p+1}(x+1)-B_{p+1}(0)}{p+1}
$$

Leo Moser proved that for another solution of the EM equation two must divide $p$, see [1], which yields that $p+1$ must be odd and $B_{p+1}(0)$ with odd subscripts is equal to zero.

Lemma 3.2.

$$
\begin{gathered}
B_{p+1}(x+1)-B_{p+1}(x)=(p+1) x^{p} \\
B_{p+1}(x+2)-B_{p+1}(x+1)=(p+1)(x+1)^{p}
\end{gathered}
$$

Proof: Relation of Bernoulli polynomials given by Whittaker and Watson, see [2], in general form is defined as $B_{n}(x+1)-B_{n}(x)=n x^{n-1}$.

Lemma 3.3. Eq. (3.1) in combination with rearranged Eq.(3.2) gives a relation

$$
\frac{B_{p+1}(x+1)}{B_{p+1}(x)}=\frac{(x+1)^{p}}{(x+1)^{p}-x^{p}}
$$

Proof: Let us express $p+1$ from Eq.(3.2) as

$$
\frac{B_{p+1}(x+1)}{x^{p}}-\frac{B_{p+1}(x)}{x^{p}}=p+1
$$

after substitution of LHS of Eq. (3.5) in Eq. (3.1) we get

$$
B_{p+1}(x+1)=(x+1)^{p}\left(\frac{B_{p+1}(x+1)}{x^{p}}-\frac{B_{p+1}(x)}{x^{p}}\right)
$$

and after elementary rearrangements we can rearrange Eq. (3.1) to the form defined in Lemma (3.3.). 


\section{Solution of the Erdös-Moser Equation}

Theorem 3.4. The EM equation has other solution than trivial if and only if holds the following equation.

$x, p \in \mathbb{N} \wedge x>2 \wedge p>1$

$$
\frac{B_{p+1}(x+2)}{B_{p+1}(x+1)}=2
$$

Proof: Let us rearrange Eq. (3.1) as

$$
B_{p+1}(x+1)=(p+1)(x+1)^{p}
$$

the RHS of Eq. (3.3) and Eq. (3.7) are equal, so we can define

$$
\begin{gathered}
B_{p+1}(x+2)-B_{p+1}(x+1)=B_{p+1}(x+1) \\
B_{p+1}(x+2)=2 B_{p+1}(x+1) \\
\frac{B_{p+1}(x+2)}{B_{p+1}(x+1)}=2
\end{gathered}
$$

Lemma 3.5. Let us define a set

$$
Z=\left\{\frac{B_{p+1}\left(x_{z}+1\right)}{B_{p+1}\left(x_{z}\right)}=\frac{\left(x_{z}+1\right)^{p}}{\left(x_{z}+1\right)^{p}-x_{z}{ }^{p}}: x_{z}, p \in \mathbb{N} \wedge p>1\right\}
$$

which contains Eq. (3.4) defined in Lemma (3.3.)

Example 3.6. $Z=\left\{\frac{B_{p+1}(1)}{B_{p+1}(0)}=\frac{(1)^{p}}{(1)^{p}-0^{p}}, \frac{B_{p+1}(2)}{B_{p+1}(1)}=\frac{(2)^{p}}{(2)^{p}-1^{p}} \ldots\right\}$.

and a set

$$
F=\left\{\frac{B_{p+1}\left(x_{f}+2\right)}{B_{p+1}\left(x_{f}+1\right)}=2: x_{f}, p \in \mathbb{N} \wedge x_{f}>2 \wedge p>1\right\}
$$

which contains all Eq. (3.6) with all possible non-trivial solutions $x_{f}$ satisfying this equation

Example 3.7. Let us assume that $x_{f}=4$ is the non-trivial solution. Then $F=$ $\left\{\frac{B_{p+1}(6)}{B_{p+1}(5)}=2\right\}$. then

$$
F \subseteq Z
$$

Remark 3.8. From the definitions of the sets in Lemma (3.5.) follows that $x_{f}$ is a variable of a corresponding element $\frac{B_{p+1}\left(x_{f}+2\right)}{B_{p+1}\left(x_{f}+1\right)}=2$ and $x_{z}$ is a variable of a corresponding element $\frac{B_{p+1}\left(x_{z}+1\right)}{B_{p+1}\left(x_{z}\right)}=\frac{\left(x_{z}+1\right)^{p}}{\left(x_{z}+1\right)^{p}-x_{z}^{p}}$.

Proof: The rules in the sets $Z$ and $F$ are sufficient to prove Lemma (3.5.) since we are seeking other solution than trivial and for $x_{f}>2 \wedge p>1$. It is more than clear that $F \subseteq Z$ since for every variable $x_{f}$ holds the following relation

$$
\forall x_{f}: x_{f}=x_{z}-1
$$




\section{David Stacha}

and the corresponding elements of the variables $x_{z}, x_{f}$, which are in relation (3.8), in both sets are equal. This finishes the proof, see Example 3.9.

Example 3.9. Similarly as in Example (3.7.), let us assume that $x_{f}=4$ would be the nontrivial solution. This example demonstrates the fact that $F \subseteq Z$, which follows from Lemma (3.5.), since the elements in both sets of corresponding variables $x_{z}, x_{f}$, which are in relation (3.8), are equal. In this case when $x_{f}=4$, according to relation (3.8) $x_{z}=5$, and the corresponding elements are equal (see below).

$\begin{array}{rrrrr}x_{z} & \text { Elements of the set } Z & x_{f} & \text { Elements of the set } F \\ & \frac{B_{p+1}\left(x_{z}+1\right)}{B_{p+1}\left(x_{z}\right)} & =\frac{\left(x_{z}+1\right)^{p}}{\left(x_{z}+1\right)^{p}-x_{z}^{p}} & & \\ 3 & \frac{B_{p+1}(4)}{B_{p+1}(3)} & =\frac{(4)^{p}}{(4)^{p}-3^{p}} & & \\ 4 & \frac{B_{p+1}(5)}{B_{p+1}(4)} & =\frac{(5)^{p}}{(5)^{p}-4^{p}} & & \\ 5 & \frac{B_{p+1}(6)}{B_{p+1}(5)} & =\frac{(6)^{p}}{(6)^{p}-5^{p}} & & \\ & \vdots & & \end{array}$

Theorem 3.10. There is no element of the set $Z$ which is equal to two for $x_{z}>2 \wedge p>1$ and because $F \subseteq Z$, the EM equation does not have any other solution than trivial.

Proof: From Lemma (3.5.) follows $F \subseteq Z$. It is clear that the elements of each set are equations. The elements of corresponding variables $x_{z}, x_{f}$, which are in relation (3.8), are equal, hence these equations must be equal as well. Let us recall that every element of the set $Z$ is defined as $\frac{B_{p+1}\left(x_{z}+1\right)}{B_{p+1}\left(x_{z}\right)}=\frac{\left(x_{z}+1\right)^{p}}{\left(x_{z}+1\right)^{p}-x_{z}{ }^{p}}$ and every element of the set $F$ is defined as $\frac{B_{p+1}\left(x_{f}+2\right)}{B_{p+1}\left(x_{f}+1\right)}=2$. Since $F \subseteq Z$ and every element of the set $F$ is equal to two, in order to prove Theorem (3.10.), it is enough to prove that no element of the set $Z$ has an integral solution, equal to two for $p>1$, since it will be in contradiction. It is trivial to see that the expression $\frac{\left(x_{z}+1\right)^{p}}{\left(x_{z}+1\right)^{p}-x_{z} p}$ has integral solutions for $x_{z}>1$ if and only if $0<p<2$. We can easily prove this statement, since by using the binomial expansion of the elements of the set $Z$ we get very useful relation

$$
\frac{B_{p+1}\left(x_{z}+1\right)}{B_{p+1}\left(x_{z}\right)}=\frac{\left(x_{z}+1\right)^{p}}{\left(x_{z}+1\right)^{p}-x_{z}^{p}}=\frac{x_{z}^{p}+p x_{z}^{p-1}+\cdots+1}{p x_{z}^{p-1}+\cdots+1}=\frac{x_{z}^{p}}{p x_{z}^{p-1}+\cdots+1}+1
$$

where is clear that $\left(p x_{z}^{p-1}+\cdots+1\right) \nmid x_{z}^{p}$ for $p>1$. In other words, there is no element of the set $Z$ which is equal to two for $p>1$, and that is in contradiction with the fact that $F \subseteq Z$. On the basis of these facts we can state that there is only a trivial solution of the 
Solution of the Erdös-Moser Equation

EM equation, when $p=1$, as it follows from the basic formula of summation $\sum_{k=0}^{x} k^{1} \equiv$ $\frac{x(x+1)}{2}=x+1 \Rightarrow \frac{x}{2}=1$, where $\mathrm{x}$ must be equal to two. All of the above-mentioned facts unconditionally prove Theorem (3.10.) and at the same time the Erdös-Moser conjecture.

Example 3.11. Let us assume that $x_{f}=4$ is the non-trivial solution. The corresponding Eq. (3.6) (after substitution $\frac{B_{p+1}(6)}{B_{p+1}(5)}=2$ ) holds for this $x_{f}$ and this Eq. (3.6) is an element of the set $F$. Since $F \subseteq Z$, and thanks to the relation (3.8), we are able to define $x_{z}=5$ and the corresponding element of the set $Z$ as $\frac{B_{p+1}(6)}{B_{p+1}(5)}=\frac{(6)^{p}}{(6)^{p}-5^{p}}$. LHS of the elements in both sets are equal so RHS must be equal as well, but there is no element of the set $Z$ which is equal to two for $p>1$, which is in contradiction, and therefore $x_{f}=4$ can not be the non-trivial solution of the EM equation.

Acknowledgement. I take this opportunity to express gratitude to reviewer for the highly professional attitude during the review process and for the constructive comments.

\section{REFERENCES}

1. L.Moser, On the diophantine equation $1^{k}+2^{k}+3^{k}+\cdots+(m-1)^{k}=m^{k}$, Scripta Math., 19 (1953) 84-88.

2. E.T.Whittaker and G.N.Watson, A course of Modern Analysis, Cambridge University Press 3rd edition, (1920) 127. 